\title{
Multifractal Detrended Cross-Correlation Analysis of Geochemical Element Concentration
}

\author{
Wan $\mathrm{Li}^{*}{ }^{* 1,2}$, Zhu Yongqian ${ }^{1}$, Deng Xiaocheng ${ }^{1,2}$ and Lin Jiaoxiu ${ }^{1,2}$ \\ ${ }^{I}$ School of Mathematics and Information Science, Guangzhou University, Guangzhou, Guangdong 510006, China \\ ${ }^{2}$ Key Laboratory of Mathematics and Interdisciplinary Sciences of Guangdong Higher Education Institutes, Guangzhou \\ University, Guangzhou, Guangdong 510006, China
}

\begin{abstract}
We use multifractal detrended cross -fluctuation analysis (MF-DXA) to investigate nonlinear behavior of geochemical element concentration, $\mathrm{Au}-\mathrm{Cu}-\mathrm{Pb}-\mathrm{Zn}-\mathrm{Ag}$, in Shangzhuang Deposit, Shandong Province, China. We find that the generalized Hurst exponent $h(q)$ and cross-correlation exponent $h_{x y}(q)$ decrease with the increase of $q$, which indicate that all element concentration series and their cross pairs exhibit multifractal phenomena. By comparing the variability of $h(q)$ and $h_{x y}(q)$, we have found that the multifractal behavior is more obvious when $q>0$ than $q<0$ for the element Au$\mathrm{Cu}-\mathrm{Pb}-\mathrm{Zn}$ and their cross pairs. These analyses, given quantitative information about the complexity of the element concentration, lead to a better understanding of the geochemical phenomena underlying mineralization process.
\end{abstract}

Keywords: Cross-correlation, detrended fluctuation analysis, geochemical element, multifractal, nonlinear.

\section{INTRODUCTION}

Tempo-spatial distribution characteristics of geochemical element distribution is the basis of metallogenic prognosis for a region. In recent decades, the distributions of geochemical elements in rocks has been intensively investigation and research to find out universal law [1]. Geochemical element distribution often shows a highly irregular structure, and exhibits scale-dependent changes in structure, and needs nonconventional statistical methods. For a better comprehension of the element grade of volatility function properties, it is necessary to ascertain the element concentration changes by investigating the structure of latency at the microscopic level $[2,3]$. Nonlinear properties of geochemical anomalies distribution have been observed in different media caused by underlying mineralization in different ways $[4,5]$. Cheng proposed that geological anomalies are usually relevant to geochemical and geophysical anomalies because of their distinct physical and chemical properties and their surroundings [6]. The distributions of geochemical elements in disseminatedveinlet gold deposit exhibit fractal and multifractal characteristics [7-9]. But, in many cases, there still exists cross correlation between different geochemical elements. Looking for long-range correlations in geochemical element concentration series and for the cross-correlations between different elements concentration sets by multifractal analysis would help to promote our understanding of the corresponding dynamics and their future evolution.

*Address correspondence to this author at the School of Mathematics and Information Science, Guangzhou University, Guangzhou, Guangdong 510006, China; Tel: 13342882560; E-mail: wanli03100@21cn.com
In order to find the multifractal features of nonstationary series, Kantelhardt et. al proposed the method of multifractal detrended fluctuation analysis (MF-DFA) [10]. Recently, a method to unveil the multifractal features of two crosscorrelated signals and higher-dimensional multifractal measures was introduced as the generalization of DCCA [11]. The higher-dimensional method was named multifractal detrended cross-fluctuation analysis (MF-DXA) [12], which has been widely used to analysing data in different fields, such as financial, sunspot and river flow fluctuations, and meteorological [13-16].

In this paper, we used MF-DXA to investigate the correlation between $\mathrm{Au}$ and $\mathrm{Cu}-\mathrm{Pb}-\mathrm{Zn}-\mathrm{Ag}$ in Shangzhuang Deposit, Shandong Province, China. Some interesting and valuable phenomena have been found.

\section{MF-DXA}

Firstly, we briefly introduce the MF-DXA [11, 12]. We suppose that there exist two tempo-spatial series $\left\{x_{i}\right\}$ and $\left\{y_{i}\right\}, i=1,2, \ldots, N$, where $N$ is the series length. Then, cumulative sum is determined for series $\left\{x_{i}\right\}$ and $\left\{y_{i}\right\}$ respectively:

$$
X(i)=\sum_{k=1}^{i}\left[x_{k}-\left\langle x_{k}\right\rangle\right], Y(i)=\sum_{k=1}^{i}\left[y_{k}-\left\langle y_{k}\right\rangle\right]
$$

where $\langle\cdot\rangle$ represents the average value. The entire series were divided into Ns $=\operatorname{int}(\mathrm{N} / \mathrm{s})$ over-lapping boxes. The length of the boxes is $s$. Local trends are evaluated by means of the $m$-th order polynomial fitting to each of segments. Then the detrended covariance is given by 
$f^{2}(s, v)=\frac{1}{s} \sum_{i=1}^{s}\left|X_{v}(i)-\tilde{X}_{v}(i) \| Y_{v}(i)-\tilde{Y}_{v}(i)\right|$

where $\tilde{X}_{v}(i)$ and $\tilde{Y}_{v}(i)$ are the fitting polynomials in segment $v$. Over all segments are averaged to gain $q$-th order detrended covariance, defined by:

$F_{x y}(s, q)=\left\{\frac{1}{N_{s}} \sum_{v=1}^{N_{s}}\left[f^{2}(s, v)\right]^{q / 2}\right\}^{1 / q}$

for $q \neq 0$

when $q=0$

$F_{0}(s, 0)=\lim _{q \rightarrow 0} F_{x y}(s, q)=\exp \left\{\frac{1}{2 N_{s}} \sum_{v=1}^{2 N_{s}}\left[\ln f^{2}(s, v)\right]\right\}$

For several scales $s$, Eqs. (2) - (4) need to be repeated. If series $\left\{x_{i}\right\}$ and series $\left\{y_{i}\right\}$ are correlated by long-range power law, then scaling relation between detrended fluctuation function $F_{x y}(s, q)$ and the size scale $s$ can be decided as

$F_{x y}(s, q) \propto s^{h_{x y}(q)}$

where $h_{x y}(q)$ is cross-correlation exponent. The $h_{x y}(q)$ can be estimated by $\log$-log plots of $F_{x y}(s, q)$ versus $s$ for each value of $q$ analysis. When $q=2$, the MF-DXA is the standard DCCA. When $\left\{x_{i}\right\}=\left\{y_{i}\right\}$, MF-DXA reduces to the classic MF-DFA, $h_{x y}(q)=h_{x x}(q)=h(q)$. Generally, $h_{x y}(q)$ depends on $q$, which indicates the behavior of multifractality. The multifractality degree is able to be quantified by $\Delta h=h_{\max }(q)-h_{\min }(q)$.

\section{RESULTS OF ANALYSIS}

\subsection{Data Acquisition and Descriptive Statistics}

Shangzhuang gold deposit is located at Wangershan fault zone of northwestern of Shandong province, China. The ore bodies occur at the hanging wall and footwall of Wangershan fault in vein irregular shapes with the NE strike NW trend, $30^{\circ}-60^{\circ}$ in dip and the lateral trending of SW. In the field, main rock types are plagioclase-metamorphic gneiss, amphibolites and granulite in Jiaodong group, and Linglong and Guojialing granites. the method of Drilling and Control Source Audio-frequency Magnetotelluric Detecting shows that Shangzhuang gold deposit is a vertical two layer structure. The upper layer is the Linglong granite. The lower is the Guojialing granite. The main ore zone is of $1800 \mathrm{~m}$ long, $2 \sim 4 \mathrm{~m}$ thick, and around $750 \mathrm{~m}$ depth $[17,18]$.

The composition of alteration rock in the central of alteration belt has the largest degree of variation, a large number of permeability fluids carry active components into the dilation space, which has significance to the further enrichment of the ore-forming element( $\mathrm{Au})$ and the remobilization of correlative elements $(\mathrm{Cu}-\mathrm{Zn}-\mathrm{Pb}-\mathrm{Ag})$.

The data of geochemical element concentration, $\mathrm{Au}-\mathrm{Cu}$ $\mathrm{Pb}-\mathrm{Zn}-\mathrm{Ag}$, were obtained from the continuous channels with $1 \mathrm{~m}$ length in Shangzhuang Deposit. These samples were assayed. The results are used for reserve calculation as well as for this study.

In order to describe dominant features of data of geochemical element concentration, a statistical analysis was performed.

We performed a Jarque-Bera (JB) test for normality. The JB statistical quantity is

$$
J B=n\left[\frac{S^{2}}{6}+\frac{(K-3)^{2}}{24}\right]
$$

where $s$ is skewness, $k$ is kurtosis, and $n$ is the sample data length. Main descriptive statistics results of the geochemical element concentration, $\mathrm{Au}-\mathrm{Cu}-\mathrm{Pb}-\mathrm{Zn}-\mathrm{Ag}$, are shown in Table 1.

According to the data in Table 1, the kurtosis values greater than 3 are display aiguille in the probability distribution. The skewness values greater than 0 are display positive skewness. The results of Jarque-Bera test show that the values of statistic are more than the significance at the 0.05 level, which indicates sample distribution departure from Gaussian normality. So we should reject null hypothesis of following a normal distribution to five samples.

Table1. Results obtained from the statistical analysis of geochemical element concentration.

\begin{tabular}{|c|c|c|c|}
\hline Element & Sample Interval & Mean (g/t) & Standard Deviation (g/t) \\
\hline \hline $\mathrm{Au}$ & {$[0.10,15.00]$} & 1.0818 & 1.3605 \\
\hline $\mathrm{Cu}$ & {$[5.00,138.60]$} & 8.8466 & 8.3782 \\
\hline $\mathrm{Pb}$ & {$[5.70,180.80]$} & 23.5422 & 13.0665 \\
\hline $\mathrm{Zn}$ & {$[5.50,202.10]$} & 30.7875 & 15.9711 \\
\hline $\mathrm{Ag}$ & {$[0.05,0.36]$} & 0.0805 & 0.0256 \\
\hline
\end{tabular}

(1-2)

\begin{tabular}{|c|c|c|c|}
\hline Element & Kurtosis & Skewness & Jarque-Bera Statistic \\
\hline \hline $\mathrm{Au}$ & 48.6248 & 6.0647 & $55148.1812^{*}$ \\
\hline $\mathrm{Cu}$ & 148.5691 & 11.2145 & $130553.1177^{*}$ \\
\hline $\mathrm{Pb}$ & 76.4542 & 7.0725 & $54865.7166^{*}$ \\
\hline $\mathrm{Zn}$ & 29.6186 & 3.687 & $20495.9535^{*}$ \\
\hline $\mathrm{Ag}$ & 35.7305 & 4.3061 & $29718.4715^{*}$ \\
\hline
\end{tabular}

Note: *Statistical significance at the $5 \%$ level.

\subsection{Cross-Correlation Test}

We used a new cross-correlation test, proposed by Podobnik et al. [19], to quantify bivariate cross-correlation between elements $\mathrm{Au}$ and $\mathrm{Cu}-\mathrm{Pb}-\mathrm{Ag}$. We suppose that there exist two discrete-series $\left\{x_{i}\right\}$ and $\left\{y_{i}\right\}$, sharing the same length $N$, and no cross correlations between them. We define the cross-correlation function: 


$$
C_{k}=\frac{\sum_{i=k+1}^{N} x_{i} y_{i-k}}{\sqrt{\sum_{i=1}^{N} x_{i}^{2}} \sqrt{\sum_{i=1}^{N} y_{i}^{2}}}
$$

The cross-correlation coefficient $C_{k}$ is a normal distribution for asymptotically large values of $N$. Then $\frac{C_{k}}{\sqrt{(N-k) / N^{2}}}$ asymptotically behaves as a standard normal distribution, with zero mean and unit variance. The sum of squares of these variables approximately follows a chisquare distribution ( $\chi^{2}$-distribution). According to the definition of $\chi^{2}$-distribution, we can obtain the crosscorrelations statistic

$Q_{c c}(m)=N^{2} \sum_{k=1}^{m} \frac{C_{k}^{2}}{N-k} \sim \chi^{2}(m)$

which is approximately $\chi^{2}(m)$ distributed, where $m$ is a degrees of freedom. If the cross-correlations test values are larger than the critical value of $\chi^{2}(m)$, then the crosscorrelations are significant. For various values $m$, we used the critical values of $\chi^{2}$-distribution at the 0.05 significance level.

For each pair of the cross original data $\left\{x_{i}\right\}$ and $\left\{y_{i}\right\}$, $\mathrm{Au} \cdot \mathrm{Cu}, \mathrm{Au} \cdot \mathrm{Pb}, \mathrm{Au} \cdot \mathrm{Zn}$ and $\mathrm{Au} \cdot \mathrm{Ag}, Q_{c c}(m)$ were computed, with $m=1,2, \ldots, 500$, using Eq. 7. All the $Q_{c c}(m)$ of $\mathrm{Au} \cdot \mathrm{Cu}, \mathrm{Au} \cdot \mathrm{Pb}, \mathrm{Au} \cdot \mathrm{Zn}$ and $\mathrm{Au} \cdot \mathrm{Ag}$, are larger than the critical values of $\chi^{2}(m)$ distribution at the 0.05 significance level (see Fig. 1). The difference between $Q_{c c}(m)$ and critical values can describe strength of crosscorrelations. However, the $Q_{c c}(m)$ test is only qualitative. Then, we applied MF-DXA method to affirm results obtained above.

\subsection{MF-DXA Analysis of Geochemical Element Concentration}

For $\mathrm{Au} \cdot \mathrm{Cu}, \mathrm{Au} \cdot \mathrm{Pb}, \mathrm{Au} \cdot \mathrm{Zn}$ and $\mathrm{Au} \cdot \mathrm{Ag}, F_{x y}(s)$ are calculated by Eqs. (1) - (5) with $q$ ranging from -5 to 5 in increments of 0.5 and $m=2$. In order to avoid the statistical error dependent on size $s$, we take $s$ from 10 to int $(N / 5)$. Fig. (2) shows the fluctuations $F_{x y}(s)$ versus $s$ for different values of $q(q=-5,-2,0,2,5)$ for three sample. The cross-correlation exponent $h_{x y}(q)$ can be obtained by observing the slope of plot $\left(\log \left(F_{x y}(s)\right), \log (s)\right)$.

In Fig. (3), the relationship between cross-correlation exponent $h_{x y}(q)$ and $q$ by MF-DXA is displayed. For comparison, we also estimate the generalized Hurst exponents $h(q)$ of time series $\left\{x_{i}\right\}$ and $\left\{y_{i}\right\}$ by MF-DFA. When $q=2$, Hurst exponent describes the persistence of autocorrelation in a separately analyzed time series. If Hurst exponent $h(2)>0.5$, the system exhibits persistent properties; if $h(2)<0.5$, it is anti-persistent. But for the cross-correlation exponent. Table 2 shows that all of the $h(2)$ are greater than 0.5 , and the $h(2)$ of $\mathrm{Zn}$ is the largest, which indicate that the geochemical elements concentration have the long-range correlation and element $\mathrm{Zn}$ has stronger than other elements. For all cross element pairs, $h(q)$ and $h_{x y}(q)$ are decreasing functions and exhibit a strong dependence on $q$ (see Fig. 3), which confirm the multifractal behavior of these series. Apparently, stronger multifractality corresponds to higher variability of $h(q)$. Moreover, the exponent $\Delta h_{x y}$ is less when $q<0$ than $q>0$, where $\Delta h_{(q<0)}=h_{\max }(q)-h(0)$ , $\Delta h_{(q>0)}=h(0)-h_{\min }(q)$. Thus, the multifractal behavior is more obvious when $q>0$ than $q<0$ for the elements $\mathrm{Au}$ $\mathrm{Cu}-\mathrm{Pb}-\mathrm{Zn}-\mathrm{Ag}$, and their cross pairs.

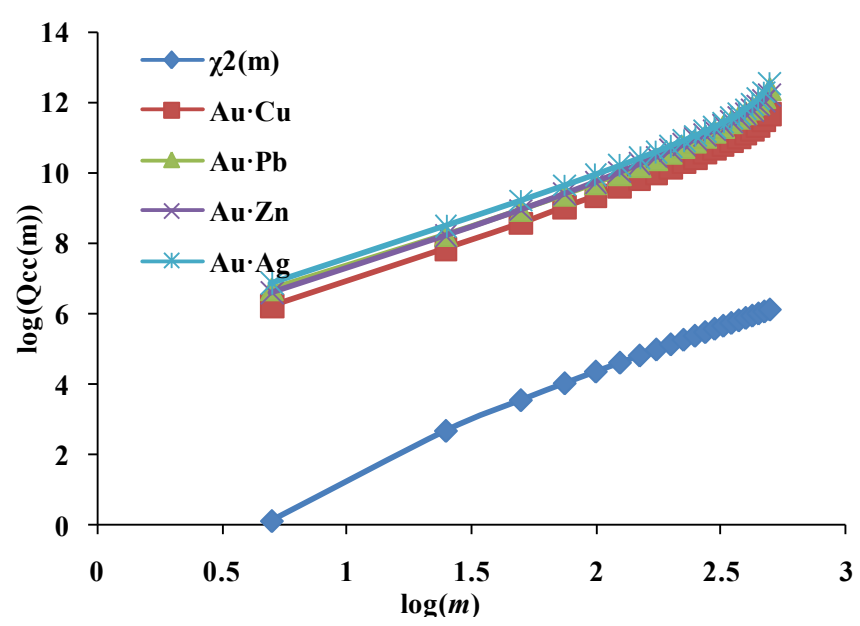

Fig. (1). $Q_{\mathrm{cc}}(m)$ versus the degrees of freedom $m$ for the $\mathrm{Au} \cdot \mathrm{Cu}$, $\mathrm{Au} \cdot \mathrm{Pb}, \mathrm{Au} \cdot \mathrm{Zn}$ and $\mathrm{Au} \cdot \mathrm{Ag}$ pairs, and for $\chi^{2}(m)$.

Table 2. The result of generalized Hurst exponents and crosscorrelation exponents.

\begin{tabular}{|c|c|c|c|}
\hline Element & $\boldsymbol{h}(\mathbf{2})$ & $\boldsymbol{\Delta} \boldsymbol{h}_{x \boldsymbol{x}(q<\mathbf{0})}$ & $\boldsymbol{\Delta} \boldsymbol{h}_{\boldsymbol{x x}(q>\mathbf{0})}$ \\
\hline \hline $\mathrm{Au}$ & 0.53 & 0.24 & 0.42 \\
\hline $\mathrm{Cu}$ & 0.62 & 0.31 & 0.87 \\
\hline $\mathrm{Pb}$ & 0.66 & 0.45 & 0.65 \\
\hline $\mathrm{Zn}$ & 0.80 & 0.11 & 0.26 \\
\hline $\mathrm{Ag}$ & 0.68 & 0.13 & 0.37 \\
\hline
\end{tabular}

(2-2)
\begin{tabular}{|c|c|c|c|}
\hline Cross-Element & $\boldsymbol{h}_{x y}(\mathbf{2})$ & $\boldsymbol{\Delta} \boldsymbol{h}_{\boldsymbol{x y}(q<\mathbf{0})}$ & $\boldsymbol{\Delta} \boldsymbol{h}_{\boldsymbol{x y}(q>\mathbf{0})}$ \\
\hline \hline $\mathrm{Au} \cdot \mathrm{Cu}$ & 0.73 & 0.20 & 0.53 \\
\hline $\mathrm{Au} \cdot \mathrm{Pb}$ & 0.66 & 0.23 & 0.51 \\
\hline $\mathrm{Au} \cdot \mathrm{Zn}$ & 0.71 & 0.15 & 0.30 \\
\hline $\mathrm{Au} \cdot \mathrm{Ag}$ & 0.70 & 0.12 & 0.25 \\
\hline
\end{tabular}



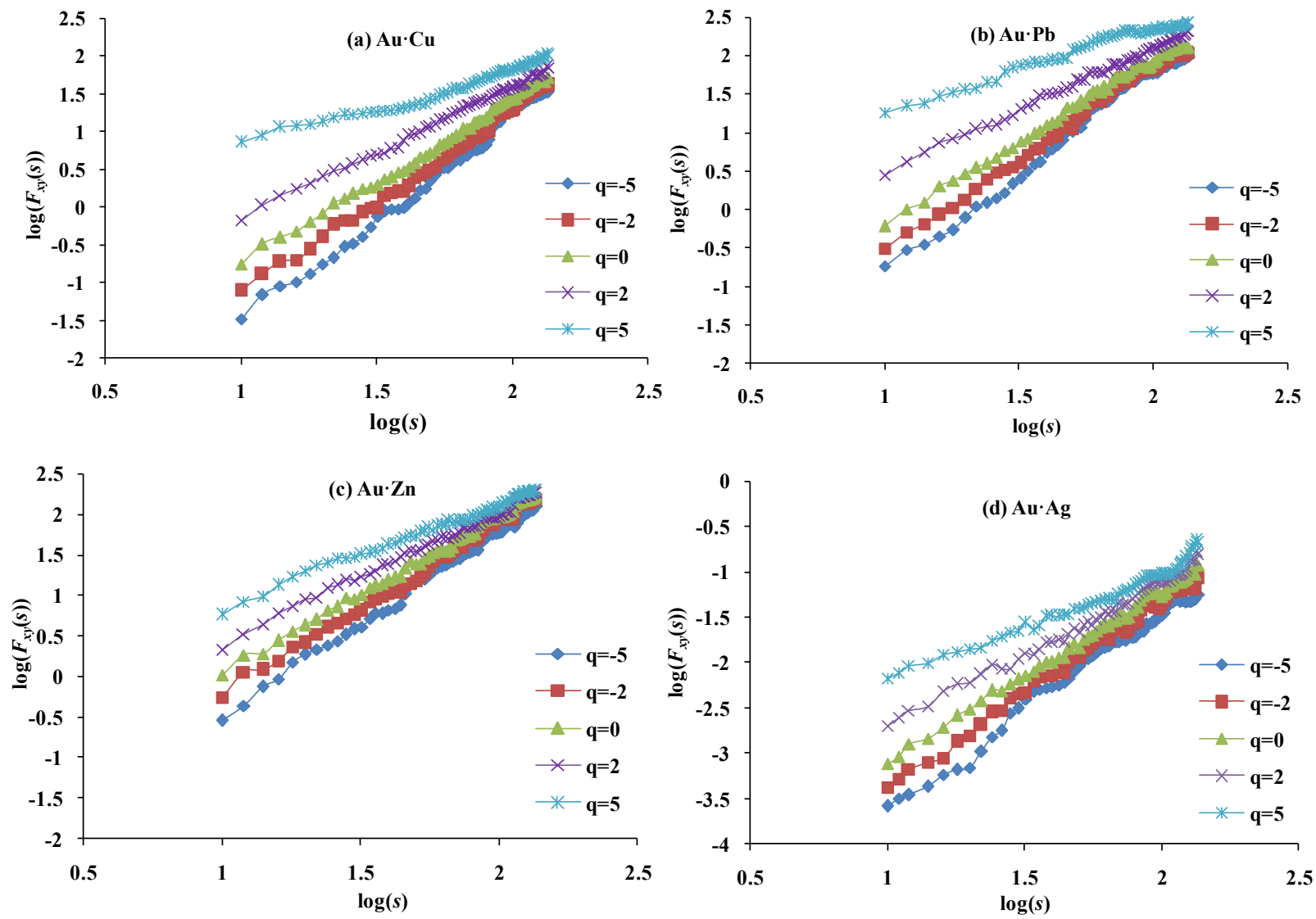

Fig. (2). The nonlinear relationships between $\log F_{x y}(s)$ and $\log (s), m=2$. (a) $\mathrm{Au} \cdot \mathrm{Cu} ;($ b) $\mathrm{Au} \cdot \mathrm{Pb} ;(\mathrm{c}) \mathrm{Au} \cdot \mathrm{Zn}$ and (d) $\mathrm{Au} \cdot \mathrm{Ag}$.
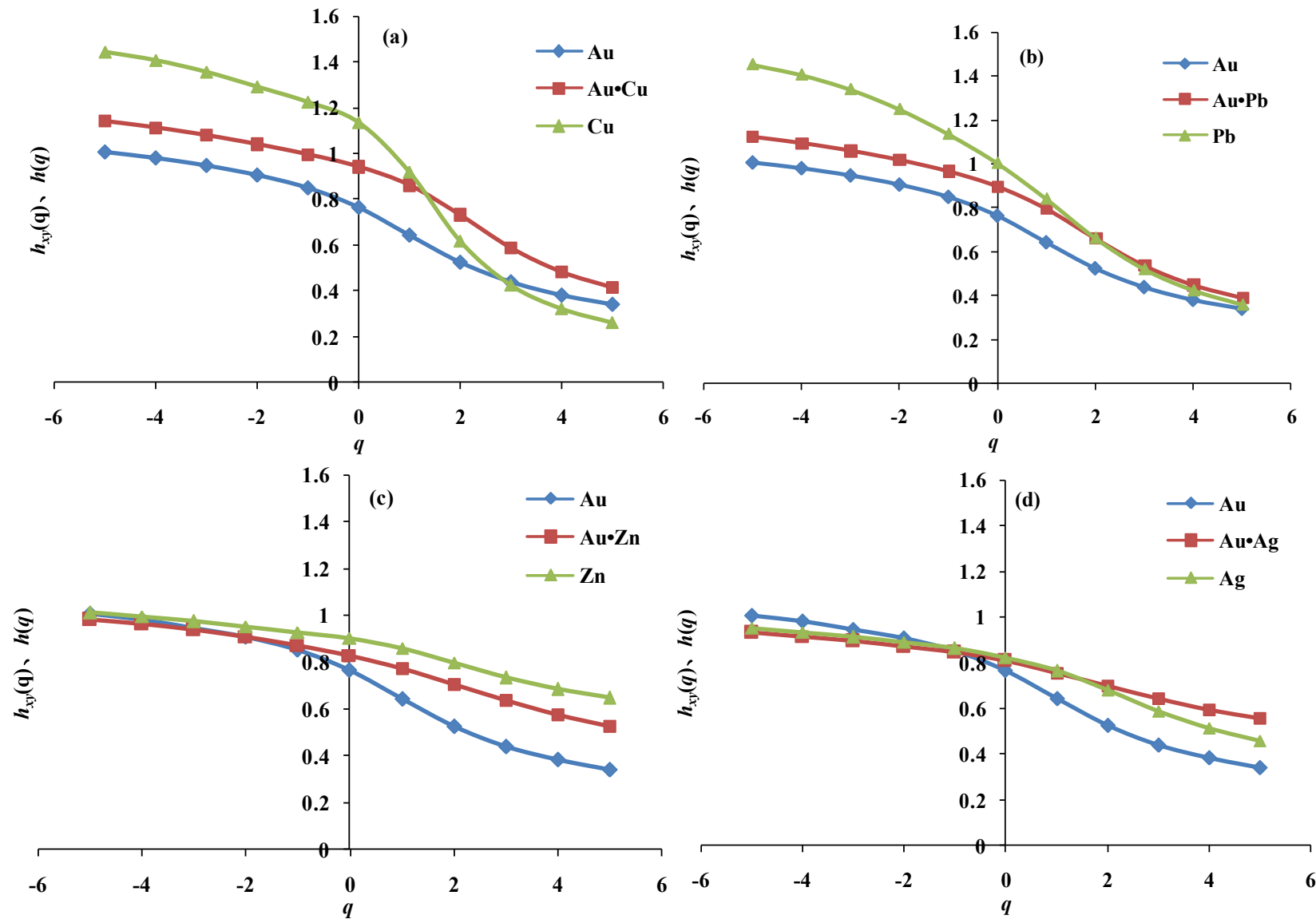

Fig. (3). Generalized scaling exponent $h(q)$ and cross-correlation exponent $h_{x y}(q)$. 


\section{CONCLUSION}

Identifying the mechanisms and interactions that influence the spatial structure of geochemical element concentration distribution is important for both metallogenic prognosis and quantitative assessment. In the paper, we have analyzed not only qualitatively for cross-correlation, but also quantitatively for the multifractal behavior of geochemical element concentration of $\mathrm{Au}-\mathrm{Cu}-\mathrm{Pb}-\mathrm{Zn}-\mathrm{Ag}$, in Shangzhuang Deposit, Shandong Province, China, by MF-DXA technique. The results show that the generalized Hurst exponent $h(q)$ and cross-correlation exponent $h_{x y}(q)$ decrease with the increase of $q$, which indicate that all element concentration series and their cross pairs exhibit multifractal phenomena. By comparing the variability of $h(q)$ and $h_{x y}(q)$, we have found that the multifractal behavior is more obvious when $q>0$ than $q<0$ for the elements $\mathrm{Au}-\mathrm{Cu}-\mathrm{Pb}-\mathrm{Zn}-\mathrm{Ag}$ and their cross pairs. These analyses, given quantitative information about the complexity of the element concentration series, lead to a better understanding of geochemical phenomena underlying mineralization process.

\section{CONFLICT OF INTEREST}

The author confirms that this article content has no conflict of interest.

\section{ACKNOWLEDGEMENTS}

This research is supported by the National Natural Science Foundation of China (Grant No. 41172295).

\section{REFERENCES}

[1] Zhu YF. Introduction Geochemistry of Mineral Deposits. Beijing: Beijing University Press 2012.

[2] Yu CW. Complexity of Geosystem. Beijing: Geological Publishing House 2007.

[3] Zuo RG, Cheng QM, Xia QL. Application of fractal models to characterization of vertical distribution of geochemical element concentration. J Geochem Explor 2009;102: 37-43.
[4] Cheng QM. Mapping singularities with stream sediment geochemical data for prediction of undiscovered mineral deposits in Gejiu, Yunnan Province, China. Ore Geol Rev 2007; 32: 314-24.

[5] Cheng QM, Agterberg FP. Singularity analysis of ore-mineral and toxic trace elements in stream sediments. Comput Geosci 2009; 35: 234-44.

[6] Cheng QM. Singularity theory and methods for mapping geochemical anomalies caused by buried sources and for predicting undiscovered mineral deposits in covered areas. J Geochem Explor 2012; 122: 55-70.

[7] Agterberg FP. Mixtures of multiplicative cascade models in geochemistry. Nonlin Process Geophys 2007; 14: 201-9.

[8] Deng J, Wang QF, Wan L, et al. A multifractal analysis of mineralization characteristics of the Dayingezhuang disseminatedveinlet gold deposit in the Jiaodong gold province of China. Ore Geol Rev 2011; 40: 54-64.

[9] Deng J, Wang QF, Wan L, et al. Self-similar fractal analysis of gold mineralization of Dayingezhuang disseminated-veinlet deposit in Jiaodong gold province, China. J Geochem Explor 2009; 102: 95-102.

[10] Kantelhardt JW, Zschiegner SA, Koscielny-Bunde E, et al. Multifractal detrended fluctuation analysis of nonstationary time series. Phys A 2002; 316: 87-114.

[11] Zhou WX. Multifractal detrended cross-correlation analysis for two nonstationary signals. Phys Rev E 2008; 77: 066211-4.

[12] Shadkhoo1 S, Jafari GR. Multifractal detrended cross-correlation analysis of temporal and spatial seismic data. Eur Phys J B 2009; 72: 679-83.

[13] Podobnik B, Stanley HE. Detrended cross-correlation analysis: a new method for analyzing two non-stationary time series. Phys Rev Lett 2008; 100: 0841021-4.

[14] He LY, Chen SP. Multifractal detrended cross-correlation analysis of agricultural futures markets. Chaos Solitons Fractals 2011; 44: 355-61.

[15] Ma F, Wei Y, Huang DS. Multifractal detrended cross-correlation analysis between the Chinese stock market and surrounding stock markets. Phys A 2013; 392: 1659-70.

[16] Hajian S, Sadegh MM. Multifractal detrended cross-correlation analysis of sunspot numbers and river flow fluctuations. Phys A 2010; 389: 4942-57.

[17] Deng J, Yang LQ, Ge LS, et al. Reasearch advances in the Mesozoic tectonic regimes during the formation of Jiaodong ore cluster area. Prog Nat Sci 2006; 16(8): 777-84.

[18] Deng J, Wang QF, Yang LQ, et al. The structure of ore-controlling strain and stress field in the Shangzhuang gold deposit in Shandong Province, China. Acta Geol Sin 2008; 82: 769-80.

[19] Podobnik B, Grosse I, Horvati D, et al. Stanley, Quantifying crosscorrelations using local and global detrending approaches. Eur Phys J B 2009; 71: 243-50. 